\title{
The Use of Sputum Dashboard and Dispatch Riders in Mitigating Delay in Sputum Samples' Processing-Evidence from an Intervention in Lagos State, Nigeria
}

\author{
Muse Olatunbosun Fadeyi ${ }^{*}$, Akinwumi Akindele"1, Adebayo Bakare ${ }^{1}$, Bolaji Ahmed ${ }^{1}$, \\ Osman El-Tayeb', Taofeekat Ali2, Yasir Othman³, Obioha Udunze1, Gbolahan Dairo1, \\ Aderonke Agbaje ${ }^{2}$
}

${ }^{1}$ Damien Foundation Belgium (Nigeria Project), Ibadan, Oyo State, Nigeria

${ }^{2}$ Institute of Human Virology Nigeria (IHVN), Abuja, Nigeria

${ }^{3}$ Hull University Teaching Hospitals NHS Trust, Hull, United Kingdom

Email: ^fadeyi.muse@dfbnigeria.org.ng

How to cite this paper: Fadeyi, M.O., Akindele, A., Bakare, A., Ahmed, B., El-Tayeb, O., Ali, T., Othman, Y., Udunze, O., Dairo, G. and Agbaje, A. (2021) The Use of Sputum Dashboard and Dispatch Riders in Mitigating Delay in Sputum Samples' Processing-Evidence from an Intervention in Lagos State, Nigeria. Journal of Tuberculosis Research, 9, 146-159.

https://doi.org/10.4236/jtr.2021.93014

Received: July 6, 2021

Accepted: August 9, 2021

Published: August 12, 2021

Copyright $\odot 2021$ by author(s) and Scientific Research Publishing Inc. This work is licensed under the Creative Commons Attribution International License (CC BY 4.0).

http://creativecommons.org/licenses/by/4.0/ (c) (i) Open Access

\begin{abstract}
The act of referring TB presumptive individuals after verbal screening to another health facility/diagnostic centres for testing often results in large loss to follow-up, owing to the many barriers people face in accessing care. The transportation of sputum samples is an efficient method of increasing access to tuberculosis (TB) diagnostics in areas where testing is not currently available. One of the major reasons for the low yield of $\mathrm{TB}$ cases in the field was delay in sputum sample transportation to diagnostic laboratories among other factors. Damien Foundation Belgium, carried out a unique sputum riders initiatives in 20 LGAs of Lagos state between Q3-2019 and Q4-2020. A total of 25 sputum dispatch riders across the 20 LGAs in the State who are monitored routinely on an electronic dashboard were engaged for sputum logistics. Therefore, this report highlights how the initiatives have contributed to increased TB case notification in a densely populated metropolitan city in Nigeria. Overall, 48,265 units of sputum samples were transported to the laboratory from the facilities $99.9 \%$ of results which were received from the laboratory by the dispatch riders. The contribution of sputum riders to the overall $\mathrm{TB}$ case detection by the project progressively increased from $9 \%$ when the intervention began to $57 \%$ as at Q4 2020. The transportation of sputum samples by motorcycle dispatch riders is an efficient method of increasing access to TB diagnostic services in communities where testing is not currently available.
\end{abstract}




\section{Keywords}

Sputum Samples Dispatch Rider, Electronic Dashboard, Sputum Logistics, Tuberculosis

\section{Introduction}

Tuberculosis (TB) is a communicable disease that is a major cause of ill health, and the leading cause of death from a single infectious agent which accounts for more than a million deaths annually [1]. TB is caused by the bacillus Mycobacterium tuberculosis, which is spread when people who are symptomatic expel bacteria into the air when they cough, sneeze or talk. TB is a disease of poverty, and economic distress, with affected persons often experiencing marginalization, stigmatization and discrimination.

The World Health Organization in December 2010 recommended the use of GeneXpert Mycobacterium Tuberculosis/Rifampicin (MTB/RIF) for TB diagnosis and detection of drug resistance [2] [3]. This was the game changer in TB diagnosis and control by reducing the time to $\mathrm{TB}$ diagnosis and linkage to treatment. GeneXpert MTB/RIF is an automated, real-time time Nucleic Acid Amplification Technology and Polymerase Chain Reaction-based equipment, with sensitivity $(97.6 \%)$ and specificity (99.8\%) higher than that of microscopy and chest X-ray [2].

The transportation of sputum samples is an efficient method of increasing access to TB diagnostics in areas where testing is not currently available. This helps to prevent the need for patients to incur avoidable additional travel costs and leads to more equity in obtaining access to TB diagnosis and care. Sputum transportation is a more cost-effective intervention compared to acquiring additional GeneXpert MTB/RIF machine and its accessories or hiring staff to provide localized TB testing. It was designed to provide universal access to rapid diagnosis of TB by linking health facilities to the existing GeneXpert network [4]. A wellorganized specimen transportation system will reduce the average time between specimen collections to the delivery of the test results to the referral laboratory/ collection facility. It will also reduce the following parameters; contamination rate, specimen rejection rate, and the turnaround time from sample collection to the delivery of results [4]. If effective, this reduces the time from the diagnosis of TB to the enrolment of patients on appropriate treatment.

Therefore, this report highlights how the introduction of sputum transportation by dispatch riders and its monitoring on the sputum dashboard contributed to increased TB case notification in a densely populated metropolitan city in $\mathrm{Ni}$ geria.

\subsection{Problem Statement}

About a quarter of the world's population is infected with TB and men, women 
and children accounted for $56 \%, 32 \%$ and $12 \%$ of the cases in 2019 respectively [1]. Africa accounted for $25 \%$ of the global proportion of TB in 2019 [1]. In Nigeria, the estimated incident for TB is about 407,000 cases occurring annually, the mortality rate is $115 / 100,000$ population [5]. Nigeria currently ranks sixth among the 30 high burden TB countries worldwide and highest in Africa with an estimated incidence rate of 219 per 100,000 population [5]. Nigeria contributed $4.4 \%$ of the global TB cases and is one of the eight countries that accounted for two-thirds of the total TB cases globally, while the 22 other countries in WHO's list of 30 high TB burden countries accounted for $21 \%$ of the total TB cases worldwide [1] [5]. According to the World Health Organisation, Nigeria is among the ten countries that account for $64 \%$ of the global gap in TB case finding; and together with India and Indonesia account for almost half of the total gap [1].

\subsection{Justification}

Nigeria adopted GeneXpert as the premier diagnostic tool for TB in 2016, and currently has 398 GeneXpert machines installed throughout the 36 states of the federation and the Federal Capital Territory [6]; despite the large number of GeneXpert machines in the country, the average TB case notification is $27 \%$ [6]. One of the major reasons for the low yield of TB cases in the field was delay in sputum sample transportation to diagnostic laboratories among other factors. The transportation of sputum in a reliable and efficient manner is essential for effective TB patients' care, allowing for faster diagnosis, early initiation of treatment, adequate patient follow-up, and improved patients' treatment outcome. As a result, the availability of a fast and reliable sputum transportation network should be a key strategy for; improving access to modern TB diagnostic tests in areas where testing is minimally available; improving the utilization rate of GeneXpert machine and; ultimately improving the yield of TB cases.

Nigeria is a developing country with a huge deficit in health infrastructure inclusive of manpower and material resources which has negatively impacted the outcome of disease management especially drug susceptible TB (DSTB) and multidrug-resistant TB (MDR TB). In line with the goal of ending TB, the current project (Global Fund (GF) sponsored TB/HIV/RSSH GRANT intervention) aimed to increase $\mathrm{TB}$ case detection, notification of $\mathrm{TB}$ cases and their linkage to treatment to a reasonable extent. However, there were reports of inadequate GeneXpert machines in the laboratories, GeneXpert cartridges, poor electricity supply, attrition among skilled laboratory scientists/technicians, as well as insufficient laboratory reagents prior to the onset of this Global Fund sponsored TB/HIV/ RSSH GRANT intervention. Despite interventions by the National Tuberculosis and Leprosy Control Programme, GF and Lagos State Ministry of Health to mitigate the challenges earlier mentioned, there still remains the challenge of inadequate number of GeneXpert machines to cater for the population [7]. The current structure in Lagos state is such that each GeneXpert site sub serves several sample collection sites as the laboratories are quite expensive to maintain [8]. 
Similarly, the option of referring presumptive individuals from facilities without diagnostic capacity to other health facilities where GeneXpert machine and laboratory technicians are available often results in high loss to follow-up among TB cases, owing to the many barriers people face in accessing care. These barriers include distance to patients' home (far distance), difficult topography (natural terrain might be unfriendly and discourage patients from seeking required medical attention) and huge financial implication (medical care is expensive). There was no baseline data about sputum transportation by dispatch riders prior to the commencement of the project in Lagos state

\subsection{Rationale}

It is well known that simply referring $\mathrm{TB}$ presumptive individuals after verbal screening to another health facility/diagnostic centers for testing often results in large loss to follow-up, owing to the many barriers people face in accessing care. Sputum transportation network is a key strategy for improving access to laboratory services and modern TB diagnostic tests for both community and facilitybased TB services. On that note, Damien Foundation Belgium, a sub recipient of TB/HIV/RSSH GRANT in Lagos State (2019-2020) engaged the services of 25 sputum dispatch riders across the 20 LGAs in the State and this was monitored routinely on an electronic dashboard.

\section{Methods}

\subsection{Study Design}

This is a descriptive analysis of the TB/HIV/RSSH GRANT data collected between Q3 2019 and Q4 2020 to evaluate the contribution of the sputum dispatch riders and sputum dashboard to tuberculosis case detection and notification in Lagos state.

\subsection{Sampling Technique}

A total population sampling survey was used where all presumptive TB patients were included in the study.

\subsection{TB/HIV/RSSH GRANT Implementation}

The intervention was carried out in the 20 LGAs of Lagos state between Q3 2019 and Q4 2020. The sputum dispatch riders were initially engaged in nine local government areas (LGAs) namely, Apapa, Ojo, Amuwo-Odofin, Alimosho, Kosofe, Ikorodu, Lagos Island, Lagos Mainland, and Ajeromi in August 2019. In December 2019, following the success of the initial engagement of sputum dispatch riders in the nine LGAs, there was a scale-up of this service to the entire 20 LGAs in the State. The State had a total of 25 dispatch riders distributed across the 20 LGAs with three riders allocated to Alimosho LGA and two riders each deployed to Ikorodu, Ajeromi and Ojo LGAs respectively, while other LGAs had one dispatch rider assigned to them. The LGAs with large landscapes beyond what one 
dispatch rider could cover efficiently were allocated extra motorcycles with dispatch riders.

Recruitment of the dispatch riders was done using the following criteria; ownership of a valid driving license, driving experience of over two years, adequate knowledge of the LGA and/or residence in the LGA, minimum of secondary school certificate, ability to read and write, ability to use an android phone/ tablet, previous cordial working relationship with superior officers and passion for the job. Thereafter, successful applicants were trained on the use of android tablet for the purpose of recording data as well as uploading data to the server, work ethics, Infection Prevention and Control while transporting biological samples, occupational safety and appropriate documentation of information.

Each dispatch rider was given a motorcycle with a $200 \mathrm{cc}$ engine capacity procured by the grant. Each rider was also provided with tools which would aid efficient sputum transportation and these included; a pair of rubber boots, knee and elbow guides for safety as well as rain coat to protect from rain. Other items given to each individual rider were; a dispatch box, sputum boxes, a reflective jacket, a back pack, an identity card, two t-shirts crested with the supporting organisations' logos which conveyed messages on tuberculosis testing (as the items served as means of identification and protection for the riders). Each rider was also given an android tablet as well as a registered mobile sim card which enabled them to upload recorded data of every sputum sample to an information technology back-end in DFB office where real-time tracking of the sputum samples was done.

These items were very important to the implementation of the sputum transportation support. The sputum boxes were used for transporting sputum from the sputum collection points in the slum areas and DOTS facilities. Two sputum boxes fitted into the dispatch box that was mounted on the motorcycles particularly for maintaining the integrity of the sputum samples. The two sputum boxes were also needed due to the high quantity of samples that were generated from the sputum collection sites. The dispatch riders communicated with all stakeholders involved in the diagnosis and treatment of TB cases using an android tablet which also enhanced communication and data reporting with all the key actors in the system.

In line with the state motorbike licensing regulations, motorcycles were registered and each allocated plate numbers. Based on the operational frequency of the motorcycles, they were serviced on a monthly basis by the riders and the needed materials such as engine oil and plugs were provided by DFB. Each motorcycle utilised about 25 litres of petrol weekly and replacements of spare parts due to wear and tear such as tyres of the motorcycles were done as necessary or at most every semester (six-monthly). The organisation ensured that the motorcycles were insured, in addition, group life insurance and group personal accident insurance were also done. In addition to their monthly allowances, communication allowances were also paid to the riders for effective data reporting both on and off the field. 


\subsubsection{Recording of Forms}

The names of presumptive TB patients were recorded in a register kept at the health facility, and from such patients, the collected sputum samples were accompanied by Sputum Examination Request forms. The dispatch rider signed the register to acknowledge the collection of the sputum samples and signed the same register again when results were returned.

\subsubsection{Training}

The sputum dispatch riders were trained on; safe driving, motorbike maintenance, biosafety measures, and how to complete recording/reporting tools. They were also mandated to possess a valid driving license and insurance.

\subsubsection{Sputum Dashboard}

It is an electronic communication system linked to the collection of sputum samples and the return of results which was properly implemented according to documented procedures. It picked and notified any delay in testing or delay in the return of results due to commodity stock out, sample rejection as well as breakdown of machines or power failures at the laboratories.

\subsubsection{Monitoring \& Evaluation of the Sputum Dashboard}

Regular mentoring, supervision and corrective actions were provided by a Medical Advisor to ensure the Standard Operating Procedures were adhered to and the proper documentation were completed. A monthly summary of all the specimen deliveries were made using the Summary Form for specimen delivery/result reporting and these were discussed at review meetings to identify any challenges and provide solutions.

\subsubsection{Ancillary Materials}

Ancillary materials required for effective sputum transportation include; recording and reporting tools, sputum cups, transportation boxes, adequate ice packs to cool sample in the sputum box, a fridge with enough space to store samples until the dispatch rider arrives at the collection facility for pick-up, adequate power supply at laboratory which could be supported with inverters or power generating set, and adequate storage facility at the laboratory to preserve the sputum samples that were not processed (dirty fridge).

\subsection{Study Population}

The study population consisted of all presumptive TB patients whose samples were collected from all health facilities and communities and thereafter shipped to the laboratories by the sputum dispatch riders between Q3 2019 and Q4 2020.

\subsection{Data Collection}

Data collection was done by the 25 sputum dispatch riders who used android tablets to fill the forms on a daily basis, and the filled forms were sent to the sputum dashboard where they were downloaded at the back-end by the Information 
Technology expert.

\subsection{Data Validation and Analysis}

In order to ensure validity of the data, only the data that were harmonized with those of the various State Tuberculosis and Leprosy Control Program Team members and Local Government Tuberculosis and Leprosy Supervisor were used in data analysis. Data was collected and checked for completeness and any error was corrected immediately. The data was downloaded and analysed using Microsoft excel package. The variables were summarized using appropriate charts and tables.

\section{Results}

Table 1 shows the intervention was across six quarters spanning from Q3-2019 till Q4-2020. Overall, 48,265 units of sputum samples were transported to the laboratory from the facilities and 48,198 units (99.9\%) of results were received from the laboratory by the dispatch riders. Overall, $99.9 \%$ of sputum samples transported had their results received at the sputum collection sites. The total positive TB cases were 3498 and they were all bacteriologically confirmed. 91.5\% of the positive $\mathrm{TB}$ cases were diagnosed using the GeneXpert machine while 8.5\% were diagnosed using Ziehl Neelsen staining for AFB. Among the cases diagnosed with GeneXpert machine (3466), 98.2\% (3402) were DSTB cases while $1.8 \%$ (64) were DRTB cases. The number of patients that needed to be tested to detect one case of TB (NNT) was 29 during the onset of the study in Q3-2019, and which progressively improved to 13 in Q3-2020 and Q4-2020.

Alimosho LGA recorded the highest number of sputum transported (6311) while Ibeju-lekki LGA recorded the lowest units of sputum transported (552) by the dispatch riders during the period under review, Figure 1.

Figure 2 shows that the highest proportions of TB cases out of the samples transported by the dispatch riders during the period under review were notified

Table 1. Quarterly movement of sputum samples across Lagos state between 2019 and 2020.

\begin{tabular}{cccccccc}
\hline INDICATORS & Q3 2019 & Q4 2019 & Q1 2020 & Q2 2020 & Q3 2020 & Q4 2020 & GRAND TOTAL \\
\hline Number of sputum samples moved & 3698 & 6678 & 10,438 & 4412 & 11,173 & 11,866 & 48,265 \\
Number of results retrieved & 2745 & 7639 & 10,446 & 4382 & 11,458 & 11,528 & 48,198 \\
Number of positive results (AFB) & - & - & 0 & 0 & 17 & 15 & 32 \\
Number of positive results (DSTB) & 126 & 477 & 686 & 411 & 835 & 867 & 3402 \\
Number of positive results (DRTB) & - & - & 13 & 17 & 21 & 13 & 64 \\
Proportion of DRTB/DSTB cases (\%) & - & - & 1.9 & 4.1 & 2.5 & 1.5 & 1.9 \\
Yield (\%) & 3.4 & 7.1 & 6.7 & 9.7 & 7.8 & 7.5 & 7.3 \\
Number needed to test & 29 & 14 & 15 & 10 & 13 & 13 & 14 \\
Error samples & 6 & 19 & 115 & 63 & 88 & 79 & 25 \\
Number of dispatch riders & 10 & 10 & 25 & 25 & 25 & 25 & 25 \\
\hline
\end{tabular}




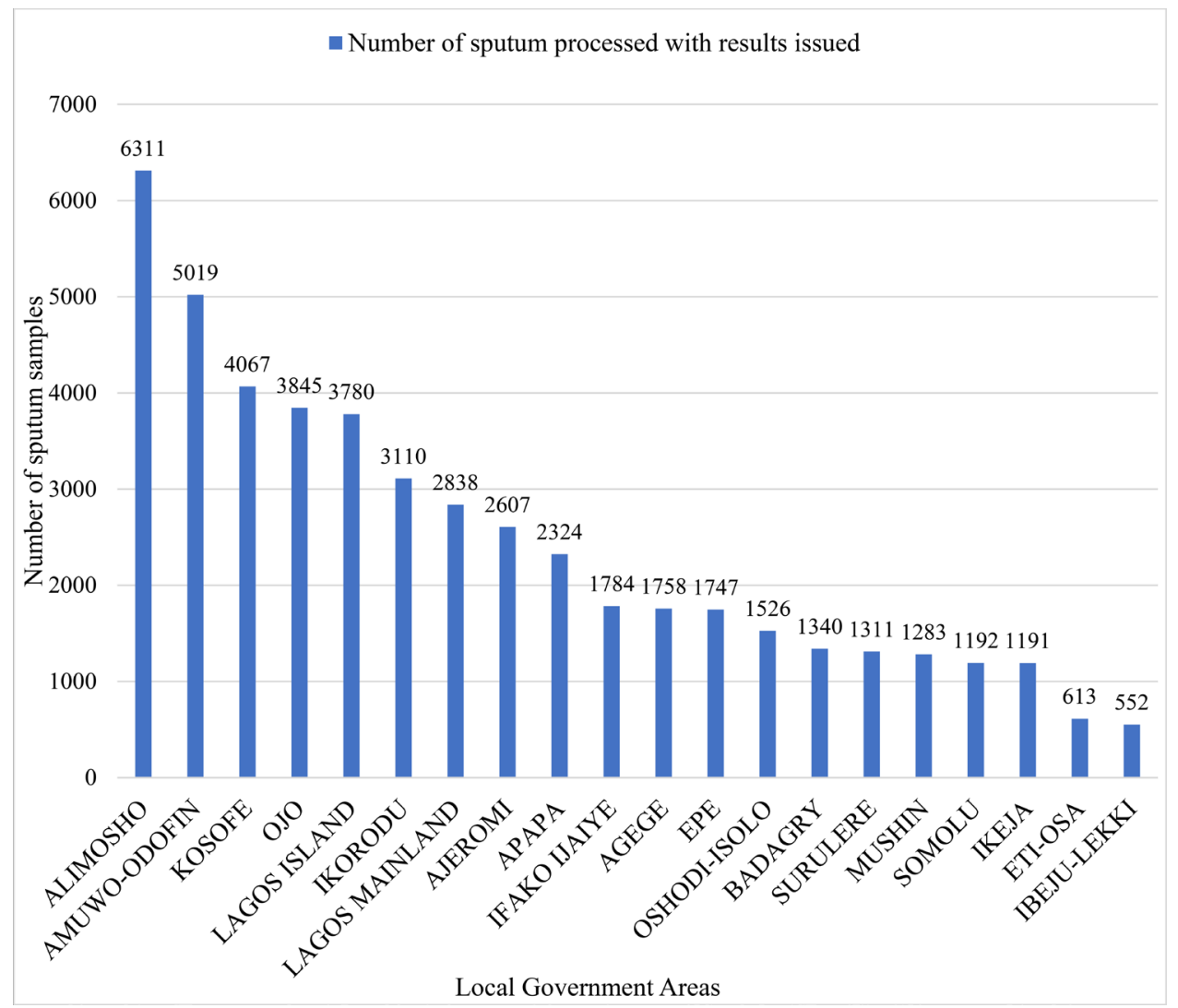

Figure 1. Sputum processed with corresponding results issued across the 20 LGAs in Lagos state.

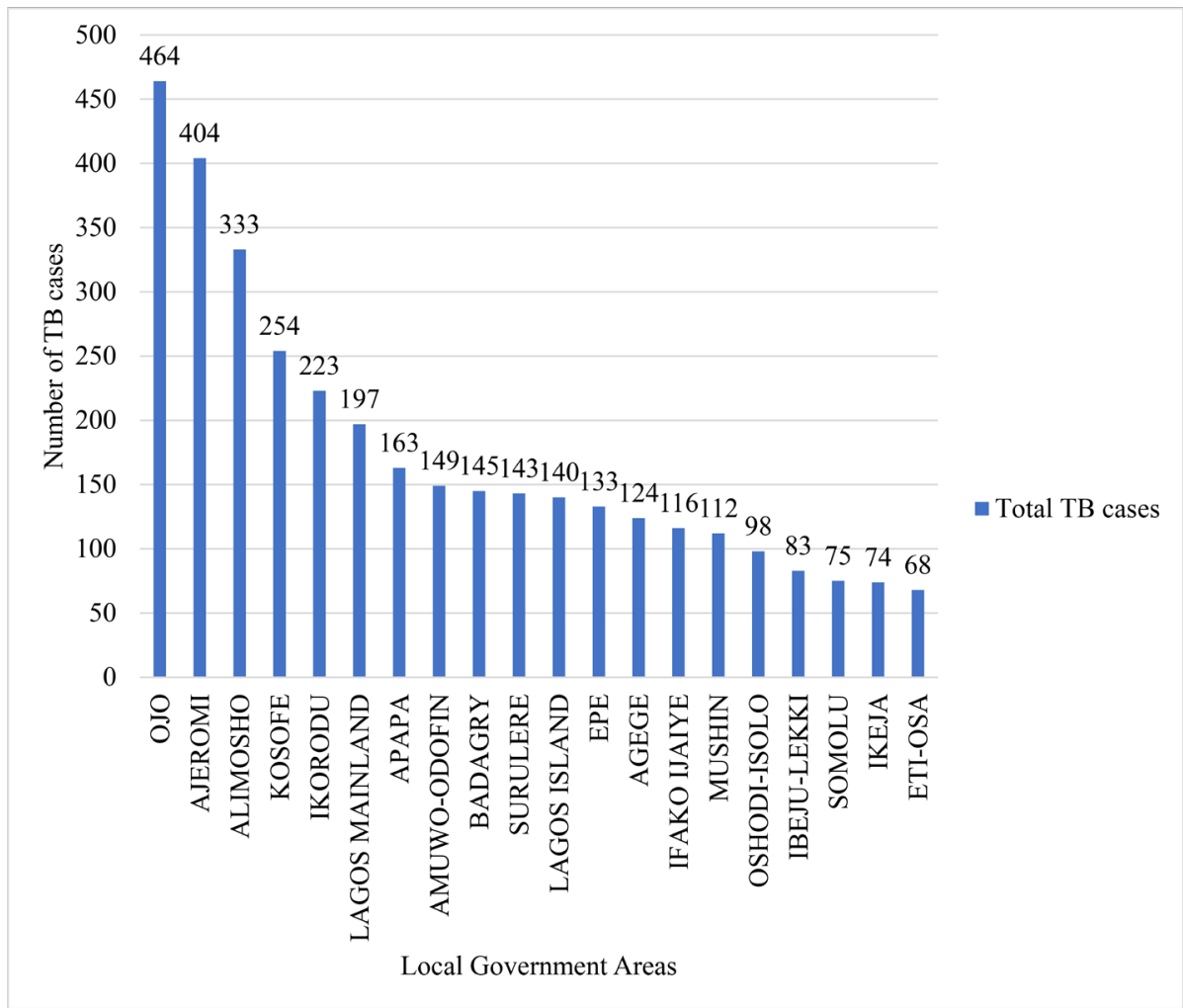

Figure 2. Tuberculosis cases identified and notified across the 20 LGAs in Lagos state 
in Ojo, Ajeromi, Alimosho, Kosofe and Ikorodu LGAs with 464 cases, 404 cases, 333 cases, 254 cases and 223 cases respectively while the lowest proportions were found in Somolu (75), Ikeja (74) and Eti-osa (68) LGAs.

There was a progressively increasing number of sputum samples transported from the facilities to the laboratories by the dispatch riders from 3698 units in Q3-2019 to 11,866 units in Q4-2020. Similarly, there was a progressively increasing number of results received from the laboratory from 2745 units in Q32019 to 11,528 units in Q4-2020. However, there was an observed dip in the volume of samples transported to the laboratory (4412 units) as well as the number of results received from the laboratory (4382 units) in Q2-2020.

Results retrieval was sub-optimal at the onset in Q3-2019, with about 74\% of results retrieved by the dispatch riders, while it was highest in Q4-2019 with $114 \%$ of results retrieved. A lower proportion of results (2745 units, 4382 units and 11,528 units) were received compared to the number of samples transported to the laboratory (3698 units, 4412 units and 11,866 units) by the dispatch riders in Q3-2019, Q2-2020 and Q4-2020 respectively. A higher proportion of results (7639 units, 10,446 units and 11,458 units) were retrieved relative to the number of samples sent to the laboratory (6678 units, 10,438 units and 11,173 units) in Q4-2019, Q1-2020 and Q3-2020 respectively, Figure 3.

The contribution of sputum transportation by dispatch riders to the TB case finding effort in Lagos state progressively increased from 126 DSTB cases in Q32019 to 867 DSTB cases in Q4-2020, while there was a dip in case finding in Q22020 with 411 DSTB cases reported, Figure 4.

Figure 5 shows that there were no DRTB cases reported in both Q3-2019 and

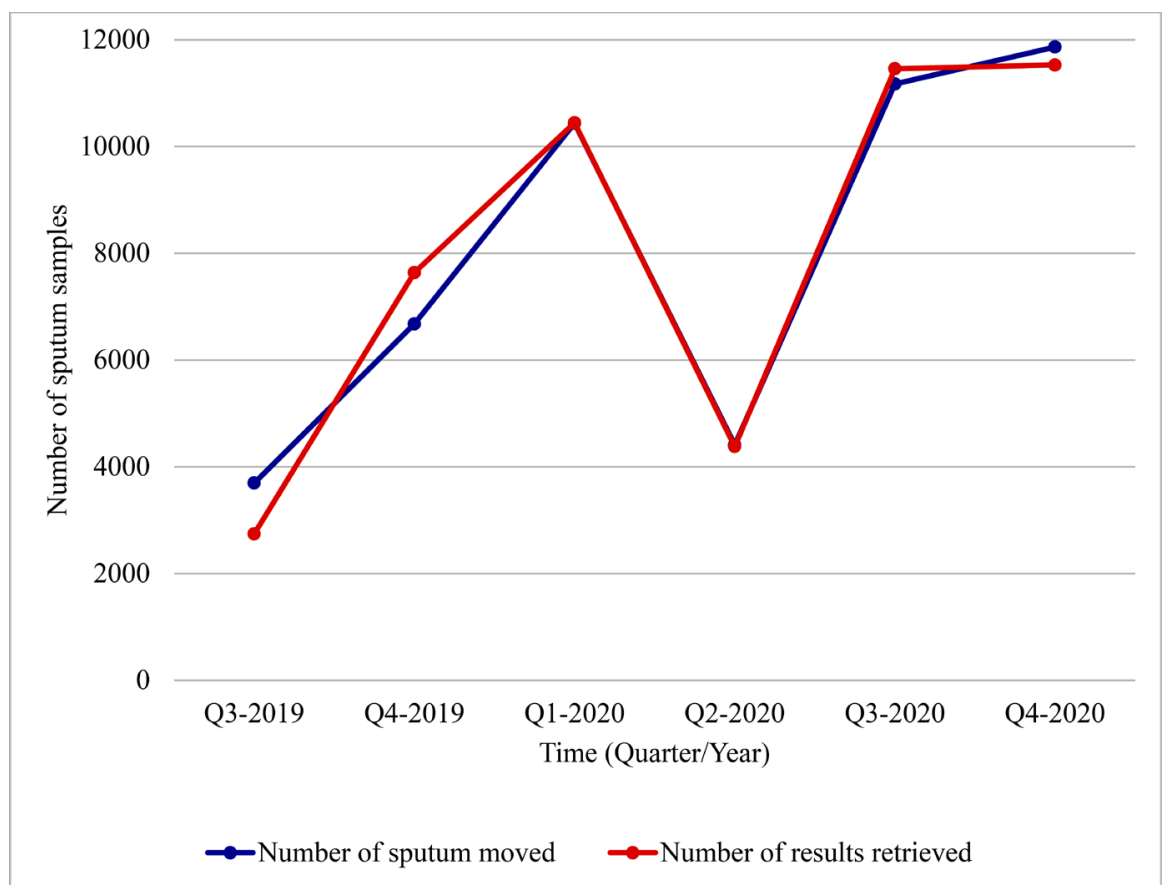

Figure 3. Quarterly transportation of sputum samples across Lagos state between Q32019 and Q4-2020. 


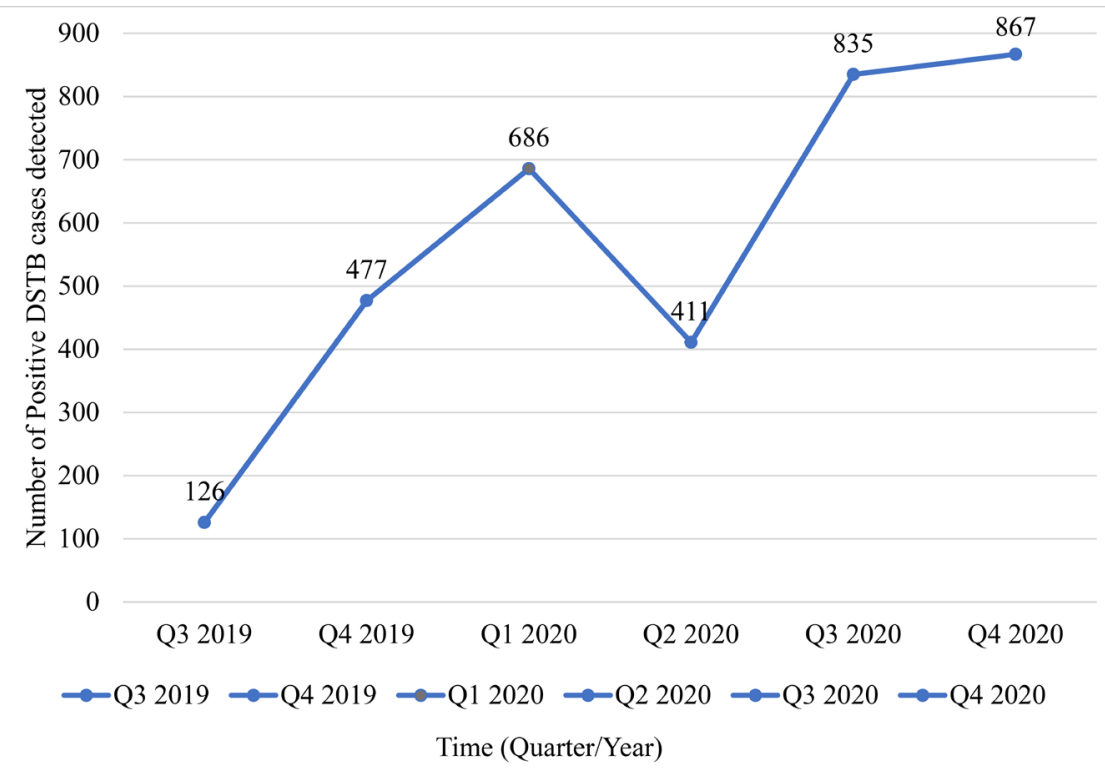

Figure 4. Positive DSTB cases detected through sputum transportation by dispatch riders in Lagos state between Q3-2019 and Q4-2020.

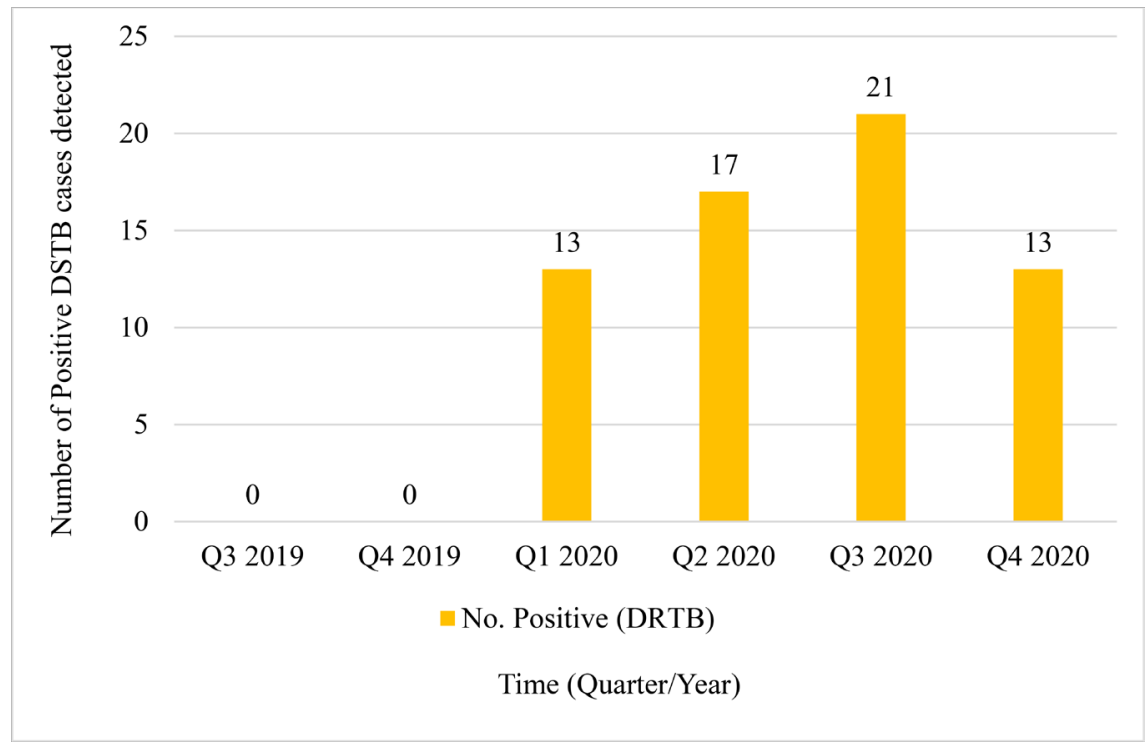

Figure 5. Positive DRTB cases detected through sputum transportation by dispatch riders in Lagos state between 2019 and 2020.

Q4-2019. The contribution of sputum transportation by dispatch riders to the case finding effort in Lagos state progressively increased from 13 DRTB cases in Q1-2020 to 21 DRTB cases in Q3-2020, while there was a dip in case finding in Q4-2020 with 13 DRTB cases reported.

The contribution of sputum riders to the overall TB case detection by the project progressively increased from $9 \%$ when the intervention began (in Q3 2019) to $57 \%$ as at Q4 2020. There was an over $500 \%$ increase in the number of TB cases diagnosed as at Q4 2020 compared to the onset of the sputum transportation by dispatch riders' intervention in Q3 2019, Table 2. 
Table 2. Contribution of dispatch riders to the overall TB cases diagnosed and notified in the project.

\begin{tabular}{cccccccc}
\hline Indicator & Q3 2019 & Q4 2019 & Q1 2020 & Q2 2020 & Q3 2020 & Q4 2020 & GRAND TOTAL \\
\hline TB case detection by the DFB in the Grant & 1339 & 1937 & 1417 & 847 & 1619 & 1566 & 8725 \\
Positive TB results from sputum transportation & 126 & 477 & 699 & 428 & 873 & 895 & 3498 \\
\% Contribution & $9 \%$ & $25 \%$ & $49 \%$ & $51 \%$ & $54 \%$ & $57 \%$ & $41 \%$ \\
\hline
\end{tabular}

\section{Discussion}

Although the project was from Q1-2019 to Q4-2020, the sputum transportation monitoring did not commence till Q3-2019 and this was due to the time lost in take-off of the implementation as the memorandum of understanding was not signed early by the stakeholders which delayed the release of funds for setting up the sputum dashboard. The sputum dashboard is an electronic monitoring board that receives real time updates about sputum transportation from the dispatch riders as they convey sputum samples from generation sites to the laboratory and also convey the results back to the originating facilities so that linkage to treatment can begin for the positive cases.

There was an observed dip in the volume of samples transported to the laboratory (4412 units) as well as the number of results received from the laboratory (4382 units) in Q2-2020, which spanned April to June 2020. Consequently, there was also a decrease in TB case finding in Q2-2020 with 411 DSTB cases reported as against 686 DSTB cases reported in the previous quarter (Q1-2020). The novel Coronavirus (COVID-19) disrupted the health system universally [9] and the Nigerian health system was not an exception. The index case of COVID-19 was first reported in Nigeria on February $27^{\text {th }}, 2020$ when an Italian travelled into the country through the Lagos international airport [9]. Afterwards, the Nigerian government implemented restrictions on gatherings and there was a lockdown declaration in Lagos state taking effect from March $30^{\text {th }}$ to July $27^{\text {th }}, 2020$ [9] [10] [11]. Lagos was locked down because of high volumes of international travels and trade through its local and international airports, sea ports and land borders [9]. This declaration (lockdown) restricted movement of people including patients from seeking healthcare in health facilities thus leading to the reduction in presumptive cases and eventual reduction in sputum samples generated for analysis at the laboratory and ultimately leading to the reduction in TB cases detected and notified [12]. Despite the reduction in sputum samples generated and transported to the GeneXpert laboratory during Q2-2020 when the lock down began in Lagos state, data show that there was an increase in the proportion of DRTB to DSTB cases from $1.9 \%$ in Q1-2020 to $4.1 \%$ in Q2-2020. This implies that the health system was greatly negatively affected by the COVID-19 lock down as there were reduced chances to diagnose new TB cases, notify and place them on treatment [12]. This is similar to the findings from a similar study in Nigeria where there was a progressive reduction in clinic attendance, presumptive TB identification, TB cases detected and treatment initiation for the TB Surge Active Case 
Finding intervention since the onset of the COVID-19 pandemic [13]. Also, there were reduced chances for TB patients and treatment supporters to access the DOTS centre for anti-TB drug refill. All these highlighted factors probably led to the spread of TB in the community leading to complications such as drug resistance and it could explain the increase in the proportion of DRTB to DSTB cases during the lockdown in Q2-2020, despite the decrease in the total number of sputum samples collected and transported to the laboratory for analysis during this period.

The yield in TB cases from the dispatch riders' intervention doubled from $3.4 \%$ at the onset of the dispatch riders' initiative in Q3-2019 compared to 7.5\% in Q4-2020, this increase implies that the initial low yield in TB cases could be attributable to the delay in transporting sputum samples to the laboratory as well as the delay in retrieval of results as there were only ten dispatch riders at the onset in Q3-2019 which was increased to 25 dispatch riders by Q1-2020. Thus, the delay in transporting sputum samples to the laboratory as well as the delay in retrieval of results was corrected by the optimisation of the dispatch riders' intervention.

The highest proportions of TB cases out of the samples transported by the dispatch riders during the period under review were notified in Ojo, Ajeromi, Alimosho, Kosofe and Ikorodu LGAs with 464 cases, 404 cases, 333 cases, 254 cases and 223 cases respectively while the lowest proportions of TB cases were found in Somolu (75), Ikeja (74) and Eti-osa (68) LGAs. The high proportions of TB cases in these five LGAs could probably be as a result of having higher numbers of motorcycle dispatch riders (all having two dispatch riders each except Ajeromi with three dispatch riders and Kosofe with one dispatch rider) compared to other LGAs in the state with one dispatch rider each. Another explanation could be that the five LGAs with the highest proportions of TB cases were among the leading eight LGAs in the state where dispatch riders conveyed the highest number of sputum samples to the laboratory for analysis with corresponding results issued. Thus, the greater the number of presumptive (symptomatic individuals) tested, the greater the number of TB cases that would likely be detected [12].

\section{Conclusion and Recommendation}

The transportation of sputum samples by motorcycle dispatch riders is an efficient method of increasing access to TB diagnostic services in communities where testing is not currently available. Evidence from this study revealed that densely populated LGAs had the highest number of TB cases reported and these include Ojo, Ajeromi, Alimosho, Kosofe and Ikorodu LGAs. Furthermore, all these LGAs had multiple motorcycles and dispatch riders except Kosofe LGA, which had one motorcycle and a dispatch rider. Thus, if Kosofe LGA could have an extra motorcycle and a dispatch rider and this can be replicated in every LGA, it could likely increase the number of TB cases reported in the LGA and ultimately 
contribute to the overall TB case detection and notification rates in the State and Country at large.

\section{Conflicts of Interest}

The authors declare no conflicts of interest regarding the publication of this paper.

\section{References}

[1] WHO (World Health Organization) (2020) Global Tuberculosis Report [Internet]. Geneva, 1-232.

https://apps.who.int/iris/bitstream/handle/10665/336069/9789240013131-eng.pdf

[2] WHO (World Health Organization) (2014) Xpert MTB/RIF Implementation Manual Technical and Operational 'How-to': Practical Considerations [Internet]. 1-52. https://apps.who.int/iris/bitstream/handle/10665/112469/9789241506700 eng.pdf?s equence $=1$

[3] WHO (World Health Organization) (2010) Roadmap for Rolling out Xpert MTB/ RIF for Rapid Diagnosis of TB and MDR-TB [Internet]. 1-12. https://www.who.int/tb/laboratory/roadmap xpert mtb-rif.pdf

[4] Izudi, J., Tamwesigire, I.K. and Bajunirwe, F. (2020) Association between GeneXpert Diagnosis and Same-Day Initiation of Tuberculosis Treatment in Rural Eastern Uganda. The American Journal of Tropical Medicine and Hygiene, 103, 1447-1454. https://doi.org/10.4269/ajtmh.19-0900 https://www.ajtmh.org/downloadpdf/journals/tpmd/103/4/article-p1447.xml

[5] NTBLCP (National Tuberculosis and Leprosy Control Programme) (2020) Bedaquiline Based All Oral Shorter Treatment Regime for DRTB Patients in Nigeria. A Modified Approach (BESTREAM). 1-38.

[6] NTBLCP (National Tuberculosis and Leprosy Control Programme) (2019) 2019 Annual TB Report [Internet]. 1-43.

https://www.health.gov.ng/doc/Draft-2019-NTBLCP-Annual-report-22032020.pdf

[7] Adepoju, P. (2020) Nigeria's Widening Tuberculosis Gap. The Lancet Infectious Diseases, 20, 29. https://doi.org/10.1016/S1473-3099(19)30712-1

https://www.thelancet.com/pdfs/journals/laninf/PIIS1473-3099(19)30712-1.pdf

[8] Abdurrahman, S.T., Emenyonu, N., Obasanya, O.J., Lawson, L., Dacombe, R., Muhammad, M., et al. (2014) The Hidden Costs of Installing Xpert Machines in a Tuberculosis High-Burden Country: Experiences from Nigeria. Pan African Medical Journal, 18, 1-5. https://doi.org/10.11604/pamj.2014.18.277.3906

https://www.researchgate.net/publication/269467251 The hidden costs of installi ng Xpert machines in a tuberculosis high-burden country experiences from $\mathrm{N}$ igeria

[9] Ilesanmi, O.S. and Afolabi, A.A. (2020) Six Months of COVID-19 Response in Nigeria: Lessons, Challenges, and Way forward. Journal of Ideas in Health, 3, 198-200. https://doi.org/10.47108/jidhealth.Vol3.IssSpecial1.63 https://www.researchgate.net/publication/344409264_Six months of COVID-19 r esponse in Nigeria lessons challenges and way forward

[10] Ilesanmi, O. and Afolabi, A. (2020) Perception and Practices during the COVID-19 Pandemic in an Urban Community in Nigeria: A Cross-Sectional Study. Peer J, 8, e10038. https://doi.org/10.7717/peerj.10038

https://www.researchgate.net/publication/344350116 Perception and practices du 
ring the COVID-19 pandemic in an urban community in Nigeria a cross-sect ional study

[11] Ibrahim, R.L., Ajide, K.B. and Olatunde Julius, O. (2020) Easing of Lockdown Measures in Nigeria: Implications for the Healthcare System. Health Policy and Technology, 9, 399-404. https://doi.org/10.1016/j.hlpt.2020.09.004

https://www.ncbi.nlm.nih.gov/pmc/articles/PMC7490626/

[12] Adewole, O.O. (2020) Impact of COVID-19 on TB Care: Experiences of a Treatment Centre in Nigeria. The International Journal of Tuberculosis and Lung Disease, 24, 981-982. https://doi.org/10.5588/ijtld.20.0418

https://theunion.org/news/impact-of-covid-19-on-tb-care-experiences-of-a-treatme nt-centre-in-nigeria

[13] Odume, B., Falokun, V., Chukwuogo, O., Ogbudebe, C., Useni, S., Nwokoye, N., et al. (2020) Impact of COVID-19 on TB Active Case Finding in Nigeria. Public Health Action, 10, 157-162. https://doi.org/10.5588/pha.20.0037

https://www.ncbi.nlm.nih.gov/pmc/articles/PMC7790486/ 